\title{
Exceptional Surface Passivation Arising from Bis(trifluoromethanesulfonyl)-Based Solutions
}

\author{
Alex I. Pointon, ${ }^{\dagger, \#}$ Nicholas E. Grant, ${ }^{\dagger, \#}$ Ruy S. Bonilla, ${ }^{\ddagger}$ Evangeline C. Wheeler-Jones, ${ }^{\|}$Marc Walker, ${ }^{\S}$ \\ Peter R. Wilshaw, Claire E. J. Dancer, " and John D. Murphy* ${ }^{*}$ \\ ${ }^{\dagger}$ School of Engineering, University of Warwick, Coventry, CV4 7AL, United Kingdom \\ ${ }^{\ddagger}$ Department of Materials, University of Oxford, Parks Road, Oxford, OX1 3PH, United Kingdom \\ ${ }^{\S}$ Department of Physics, University of Warwick, Coventry, CV4 7AL, United Kingdom \\ "Warwick Manufacturing Group, University of Warwick, Coventry, CV4 7AL, United Kingdom
}

\author{
Supporting Information
}

ABSTRACT: The surface properties of many inorganic electronic materials (e.g., $\mathrm{MoS}_{2}, \mathrm{WSe}_{2}, \mathrm{Si}$ ) can be substantially modified by treatment with the superacid bis(trifluoromethane)sulfonimide (TFSI). Here we find more generally that solutions based on molecules with trifluoromethanesulfonyl groups, including TFSI, give rise to excellent room temperature surface passivation, with the common factor being the presence of $\mathrm{CF}_{3} \mathrm{SO}_{2}$ groups and not the solution's acidity. The mechanism of passivation comprises two effects: (i) chemical passivation; and (ii) field effect passivation from a negatively charged thin film likely to be physically adsorbed by the surface. Degradation of surface passivation is caused by de-

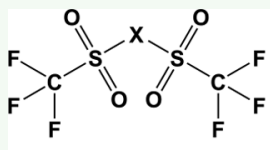

Central group $(\mathrm{X})$

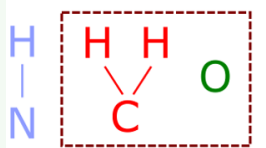

Nonacidic

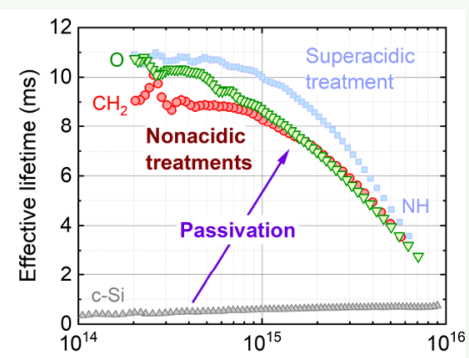

Excess carrier density $\left(\mathrm{cm}^{-3}\right)$ adsorption of the thin film from the surface, and occurs slowly in air and rapidly upon vacuum exposure. The air stability of the passivation is enhanced by the presence of droplets at the surface which act to protect the properties of the film. The finding that nonacidic solutions can provide excellent electrical passivation at room temperature opens up the possibility of using them on materials more sensitive to an acidic environment.

KEYWORDS: surface passivation, electrical passivation, superacid, recombination, luminescence, lifetime

\section{INTRODUCTION}

Ionic solutions formed from chemicals with trifluoromethanesulfonyl $\left(\mathrm{CF}_{3} \mathrm{SO}_{2}\right)$ groups have recently been shown to provide remarkable effects when they are used to treat the surfaces of a range of inorganic materials. For example, the superacid bis(trifluoromethane)sulfonimide (TFSI) has been shown to enhance photoluminescence (PL) from 2D transition metal dichalcogenides ${ }^{1}$ and quantum dots, ${ }^{2}$ to control the stability of anodes in lithium-ion batteries, ${ }^{3}$ and to provide excellent passivation of the surfaces of silicon for photovoltaic cells. $^{4-6}$ For a simple room temperature process the effects which occur are astonishing, with quantum yields from $\mathrm{MoS}_{2}$ improving from $<1 \%$ to $>95 \%{ }^{7}$ and with surface recombination velocities achieved for a passivated crystalline silicon surface of $<1 \mathrm{~cm} / \mathrm{s}^{5,6}$ being similar to the best dielectric passivation schemes. ${ }^{8}$

The superacidic properties of a TFSI-containing solution arise from the particularly labile hydrogen on the TFSI molecule, ${ }^{9}$ and effects arising from superacid-solution treatments are often explained in terms of protonation. ${ }^{10}$ For instance, it is proposed that PL enhancement in $\mathrm{MoS}_{2}$ arises from hydrogen passivation of the sulfur vacancy. ${ }^{11}$ It is also proposed that surface passivation of crystalline silicon arises from protonation, ${ }^{4}$ in which case the mechanism could essentially be the same as for liquid HF passivation. ${ }^{12}$ It has also been suggested that the TFSI molecules can diffuse into encapsulating polymers to enhance the stability of the passivation of $\mathrm{MoS}_{2}$ surfaces. ${ }^{13}$ Further to this, we have argued previously that TFSI binding to the surface is inconsistent with the dangling bond density required to explain the low surface recombination velocities achieved on crystalline silicon. ${ }^{6}$

While the benefits of TFSI-based surface treatments are clear, the mechanisms by which the effects arise are not well established. In this paper we perform a series of experiments to understand the origin of the passivation. We demonstrate that excellent surface passivation can arise from a whole family of molecules with $\mathrm{CF}_{3} \mathrm{SO}_{2}$ groups, and while some have labile hydrogen (e.g., TFSI) others do not, indicating that (super)acidity is not required for excellent passivation. This opens up the possibility of using such solutions on materials susceptible to acid damage, such as perovskite solar cells which have recently been found to benefit considerably from passivation treatments. ${ }^{14}$ We conduct experimental work to assess the contribution of field effect passivation from charges in the

Received: April 24, 2019

Accepted: June 13, 2019

Published: June 13, 2019 


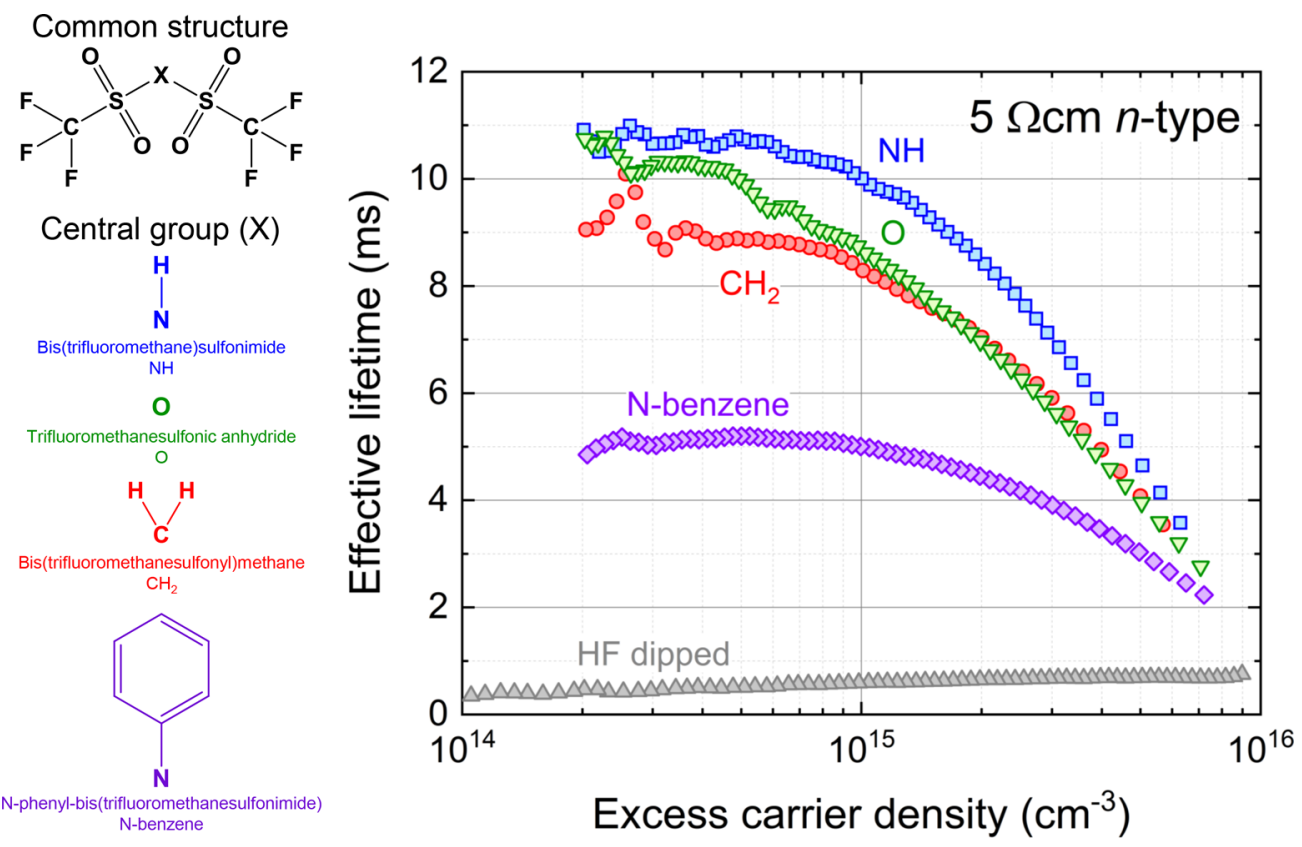

Figure 1. (Left) The common structure of the molecules in the passivating solutions investigated, with the central group of the molecule varying (designated by "X"). (Right) Lifetime measurements performed in the high-specification glovebox on high-purity silicon wafers treated with different solutions in the same glovebox. The lifetime in a control sample which was only HF-dipped to have a hydrogen terminated surface is also shown.

passivating film, finding the films to contain negative charge. We also link the stability of the passivation to residue on the sample's surfaces, and show the films have limited vacuum stability which inhibits their reliable study with high vacuum techniques.

\section{EXPERIMENTAL SECTION}

Samples and Surface Preparation. Samples were high-quality commercial $100 \mathrm{~mm}$ diameter (100) orientation $5 \Omega \mathrm{cm} n$-type silicon wafers grown by the float-zone (FZ) process. Lifetime measurements were made on quarter wafers which are sufficiently large to avoid strong edge recombination effects. ${ }^{15}$ Prior to chemical treatment, samples were subjected to a carefully optimized surface preparation process ${ }^{5,6}$ comprising an $\mathrm{HF}(1 \%)$ dip, standard clean $2\left(\mathrm{H}_{2} \mathrm{O}, \mathrm{H}_{2} \mathrm{O}_{2}\right.$ (30\%), $\mathrm{HCl}(37 \%)$ in 5:1:1 ratio) for $10 \mathrm{~min}$ at $\sim 75{ }^{\circ} \mathrm{C}$, an $\mathrm{HF}(1 \%)$ dip, an etch in $25 \%$ tetramethylammonium hydroxide (TMAH) for 10 min at $\sim 80^{\circ} \mathrm{C}$, another HF (1\%) dip, a second standard clean 2 as previously, followed by immersion in $2 \%$ HF. Samples are then pulled dry from the HF solution. After surface preparation, samples were 700 to $740 \mu \mathrm{m}$ thick.

Passivation and Lifetime Characterization. Precursor chemicals were handled and stored in a high specification glovebox (a sealed filtered MBRAUN UNIlab modular glovebox workstation) with gas purification system and solvent filter with a controlled low humidity atmosphere $\left(<0.1 \mathrm{ppm}\right.$ of $\mathrm{O}_{2} ;<0.1 \mathrm{ppm}$ of $\left.\mathrm{H}_{2} \mathrm{O}\right)$. Bis(trifluoromethane)sulfonimide (TFSI or $\mathrm{NH}$ ), trifluoromethanesulfonic anhydride (O), bis(trifluoromethanesulfonyl)methane $\left(\mathrm{CH}_{2}\right)$, and $\mathrm{N}$-phenyl-bis(trifluoromethanesulfonimide) (N-benzene) were obtained from Sigma-Aldrich. They were made into solutions with anhydrous $n$-hexane (95\% pure) or 1,2-dichloroethane (DCE, 99.8\% pure) from Sigma-Aldrich. Silicon samples were immersed in the solutions for $60 \mathrm{~s}$ at room temperature before being removed and allowed to dry. This immersion process took place in either the high specification glovebox or a lower specification glovebox with ambient flowing nitrogen (relative humidity $<25 \%$ ). Transient photoconductance decay (PCD) lifetime measurements were performed at room temperature using Sinton WCT-120 lifetime testers which were located either within the high specification glovebox or in air.
NMR. The solutions were studied in their as-prepared states by nuclear magnetic resonance (NMR) with ${ }^{1} \mathrm{H}$ NMR or ${ }^{19} \mathrm{~F}$ NMR spectra recorded on a Bruker Avance III HD 400 at 400 or $376 \mathrm{MHz}$, respectively. Sample tubes were filled in dry nitrogen atmospheres. Chemical shifts $(\delta)$ are quoted in ppm using tetramethylsilane (TMS, $\delta \mathrm{H} 0.00 \mathrm{ppm})$ as an internal reference in deuterated chloroform. For ${ }^{19} \mathrm{~F}$ NMR, $\alpha, \alpha, \alpha$-trifluorotoluene $(\delta \mathrm{F},-63.72 \mathrm{ppm})$ was added as a reference. ${ }^{19} \mathrm{~F}$ NMR is presented as hydrogen coupled.

Kelvin Probe. The surface potential was measured using a KP Technologies KP020 Kelvin probe (KP) instrument using the method of Baikie et al. ${ }^{16}$ with a gold tip and an accuracy of $\pm 50 \mathrm{mV}$. For illumination tests an LED array was used to photogenerate charge carriers near the silicon's surface. The LEDs had two broadband emission peaks at 464 and $550 \mathrm{~nm}$ wavelengths to produce white light, and the total intensity measured on the sample was $\sim 24 \mu \mathrm{W} /$ $\mathrm{cm}^{2}$.

XPS. Photoelectron spectroscopy data were collected at the Warwick Photoemission Facility using a Kratos Axis Ultra DLD spectrometer. Treated samples were mounted on to copper stubs using electrically conductive carbon tape and transferred to the spectrometer from a glovebox under inert atmosphere. The HFdipped sample was loaded into a nitrogen-purged transfer container in a cleanroom (not a glovebox). The base pressure of the XPS spectrometer was approximately $1 \times 10^{-10} \mathrm{mbar}$ and samples were pumped to below $1 \times 10^{-6} \mathrm{mbar}$ in the load lock before transfer. XPS measurements were performed using a monochromated $\mathrm{Al} \mathrm{K} \alpha \mathrm{X}$-ray source. The measurements were conducted at room temperature and at a takeoff angles of $15^{\circ}$ and $90^{\circ}$ with respect to the surface parallel. The core level XPS spectra were recorded using a pass energy of 20 $\mathrm{eV}$ (resolution approximately $0.4 \mathrm{eV}$ ) from an analysis area of $300 \mu \mathrm{m}$ $\times 700 \mu \mathrm{m}$. The spectrometer work function and binding energy scale were calibrated using the Fermi edge and $3 \mathrm{~d}_{5 / 2}$ peak recorded from a polycrystalline $\mathrm{Ag}$ sample prior to the commencement of the experiments. Fitting procedures to extract peaks positions and relative stoichiometries from the XPS data were carried out using the Casa XPS software suite, using Shirley backgrounds and mixed GaussianLorentzian (Voigt) line shapes. To test the effects of vacuum exposure, the treated silicon sample was placed in a cryostat chamber at room temperature which was evacuated. For this test, pumping down the chamber took $10 \mathrm{~min}$ to reach $<6 \times 10^{-5} \mathrm{mbar}$, the sample 

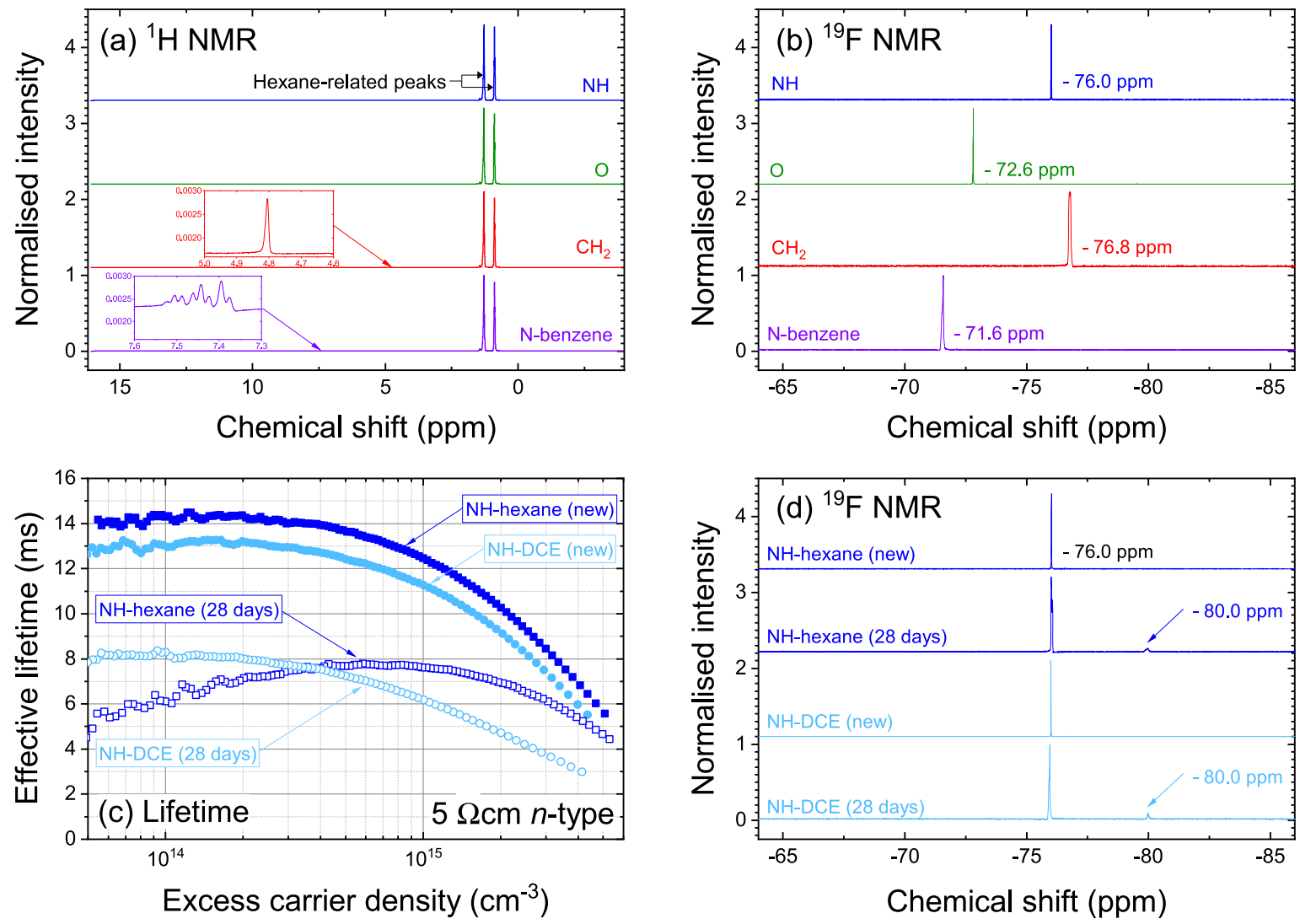

Figure 2. (a) Proton NMR and (b) fluorine NMR performed on a range of passivating solutions formed from the molecules in Figure 1 dissolved in hexane. (c) Lifetime results for $5 \Omega \mathrm{cm} n$-type silicon treated with new and 28 days' old NH(TFSI) dissolved in hexane or DCE. (d) Fluorine NMR results for the solutions used in panel c showing the appearance of an additional fluorine-related shift due to degradation of the solutions.

was held at $<6 \times 10^{-5} \mathrm{mbar}$ for $5 \mathrm{~min}$, and the chamber was vented for 7.5 min before it was opened.

Raman. Raman experiments were performed at room temperature using a Renishaw inVia Reflex Raman Microscope in confocal mode using a $532 \mathrm{~nm}$ excitation laser at $100 \%$ power $(18 \mathrm{~mW})$ through a $50 \times$ magnification lens. The point spectra data shown are summed over 10 acquisitions of $1 \mathrm{~s}$ each. Data were acquired with the Renishaw WiRE 3.1 software package.

\section{RESULTS AND DISCUSSION}

Experiments were performed to passivate surfaces with different solutions formed by molecules with a bis(trifluoromethanesulfonyl)-based structure. For these experiments hexane was used as the solvent as it has been previously shown to provide better stability ${ }^{6}$ than 1,2-dichloroethane (DCE) used in other studies. ${ }^{1,4,5}$ The molecules are shown in Figure 1, and have a common structure, except with different functional groups in the position marked " $\mathrm{X}$ " in the center of the molecule. They are labeled according to the " $X$ ", with bis(trifluoromethane)sulfonimide (TFSI) designated "NH", trifluoromethanesulfonic anhydride designated "O", bis(trifluoromethanesulfonyl)methane designated " $\mathrm{CH}_{2}$ ", and $\mathrm{N}$ phenyl-bis(trifluoromethanesulfonimide) designated "N-benzene".

Minority carrier lifetime measurements made for $5 \Omega \mathrm{cm} n$ type silicon treated with the different solutions at room temperature are shown in Figure 1. Also shown is an HFdipped sample which was subjected to the same cleaning procedures as the other samples but no additional passivation treatment. The lifetime in the HF-dipped sample is substantially higher than a free silicon surface as the silicon bonds are temporarily terminated/passivated by hydrogen. ${ }^{12}$ Room temperature treatment with all four solutions results in high effective lifetimes due to improved surface passivation. Excellent surface passivation from TFSI (labeled "NH" here) has been found for silicon ${ }^{4-6}$ and other materials ${ }^{1,2,7,10,13,17,18}$ previously, and it is frequently referred to as "superacid passivation". Importantly, the data in Figure 1 demonstrate that the passivation effect arises more generally than with just a superacid. Other molecules which do not have labile hydrogen $\left(\mathrm{O}, \mathrm{CH}_{2}\right.$, and $\mathrm{N}$-benzene) but have a similar molecular structure to TFSI $(\mathrm{NH})$ give excellent surface passivation as well. This suggests that the superacidic properties (i.e. high ionic hydrogen content) are not the reason for the exceptional passivation found previously for TFSI $(\mathrm{NH})$.

The passivating solutions (bis(trifluoromethanesulfonyl)based molecules in hexane) were studied by nuclear magnetic resonance (NMR). Proton NMR results in Figure 2a show a common pair of peaks arising from the hexane solvent. Weak signals (shown as insets) appear for the $\mathrm{CH}_{2}$ and N-benzene solutions. No such weak signal was found for the $\mathrm{NH}$ solution due to the labile central hydrogen in this superacidic solution. Results from ${ }^{19} \mathrm{~F}$ NMR are presented in Figure $2 \mathrm{~b}$, with peaks found in the -71 to $-77 \mathrm{ppm}$ range as expected for trifluoromethyl $\left(\mathrm{CF}_{3}\right)$ groups. ${ }^{19}$ Our measured values agree well to the previously measured values for $\mathrm{NH}$ (TFSI) of $-76.0 \mathrm{ppm}^{20}$ and $\mathrm{O}$ of $-72 \mathrm{ppm}^{19}$ Our results are also consistent with the known trend of the shift becoming more negative as the charge on the $\mathrm{CF}_{3} \mathrm{SO}_{2}$ increases. ${ }^{20}$ Importantly, no other shifts are found in the fluorine NMR spectra than those shown in Figure $2 b$, which suggests no initial 
(a)

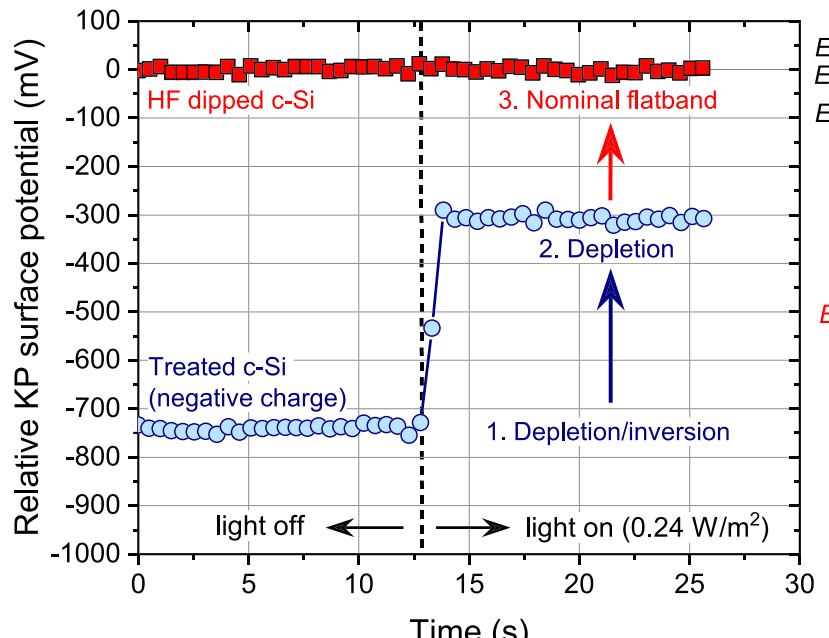

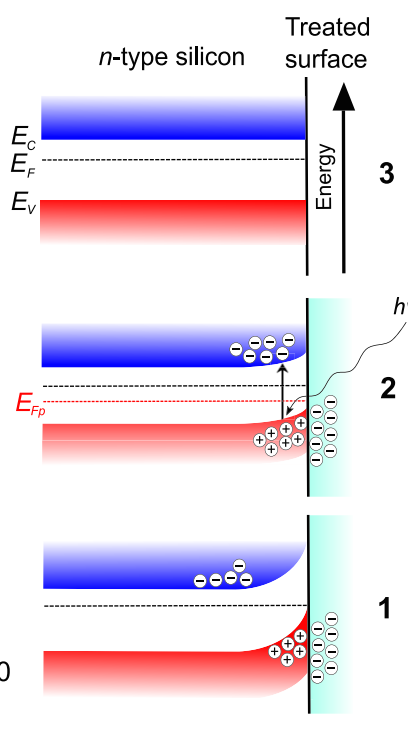

3

$h v$

2

1
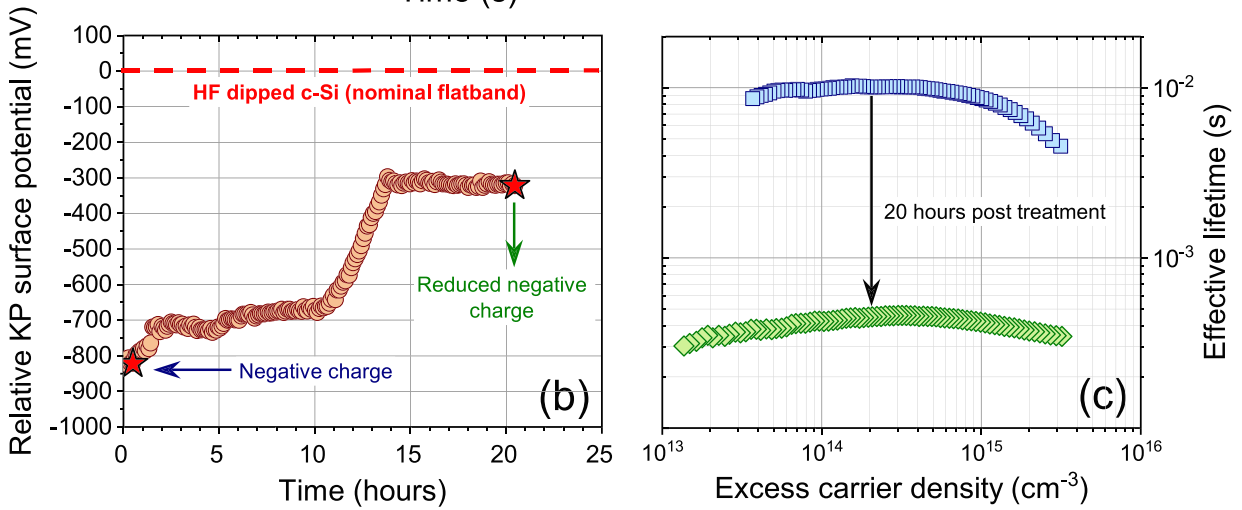

Figure 3. Experiments to establish the role of field effect passivation on $5 \Omega \mathrm{cm} n$-type silicon surfaces treated with NH(TFSI)-hexane. (a) Kelvin probe (KP) measurements (left) show the relative effect of LED illumination on the surface potential for treated and HF-dipped samples, demonstrating that the passivating film is negatively charged. The band diagrams (right) illustrate the effects of illumination on the surface band structure. (b) Relative KP surface potential over an extended time period. (c) Lifetime measurements made in air on the same sample used for KP measurements in panel $b$ showing the reduction in lifetime which has occurred.

decomposition of TFSI in hexane affecting the fluorine atoms. This does not necessarily mean that the chemical passivation of the silicon surface arises from the intact molecules, which seems unlikely due to molecular sterics and the number of terminated bonds as we have argued previously. ${ }^{6}$ Studies ${ }^{3,21}$ have proposed mechanisms for the decomposition of the TFSI anion, and it is possible that the anion breaks-up in the vicinity of the silicon surface. In such a case, a component of the bis(trifluoromethanesulfonyl)-based molecule (e.g., $\mathrm{CF}_{3}$ or $\mathrm{SO}_{2} \mathrm{CF}_{3}$ ) could play a role in chemically passivating the surface. However, it is also possible that the chemical passivation arises mainly from hydrogen termination from the HF dip immediately prior to the treatment in the bis(trifluoromethanesulfonyl)-based solution, and that the film formed from the solution stabilizes the chemical passivation.

The surface passivation level achieved with TFSI (NH) can depend on the solvent used, ${ }^{4,6}$ with passivation also affected by the age of the solution used. ${ }^{6}$ Lifetime results in Figure $2 \mathrm{c}$ show superior passivation results with new solutions in hexane compared to 1,2-dichloroethane (DCE) as used in previous passivation studies of silicon. ${ }^{4,5}$ For solutions aged for 28 days, the hexane solution generally gives better results than the DCE solution, with the latter also experiencing a stronger injection- dependence. ${ }^{19} \mathrm{~F}$ NMR results in Figure $2 \mathrm{~d}$ show the presence of an additional peak in both aged solutions with a shift of $-80.0 \mathrm{ppm}$. This shift is in the range expected for a derivative of trifilic acid, ${ }^{19}$ and so we attribute this to a $\mathrm{CF}_{3} \mathrm{SO}_{2}$ ion which has originated from the TFSI molecule which has degraded in the presence of the solvent. This is evidence for the passivating solutions degrading over time. A series of NMR experiments done over time (see Supporting Information, Figure S1) show the -80.0 ppm peak to become detectable by NMR between 7 and 14 days for DCE and 14 and 28 days for hexane. It is noted that the DCE solution visibly changes color after 28 days but the hexane solution does not (see Figure S2). A similar injection-dependence in the aged DCE lifetime in Figure 2c was previously observed with TFSI (NH) in acetone, which also undergoes a color change. ${ }^{6}$

In general, the passivation of surfaces occurs by chemical passivation (i.e., termination of dangling bonds) and/or by field effect passivation whereby charge in a layer repels carriers away from the surface. Kelvin probe $(\mathrm{KP})$ measurements were used to determine whether charge plays a role in our passivation process. Figure 3 a shows the surface potential of a TFSI-hexane treated silicon surface and an HF-dipped $n$ type silicon surface. Illumination of the HF-dipped $n$-type surface does not change the surface potential, whereas the 

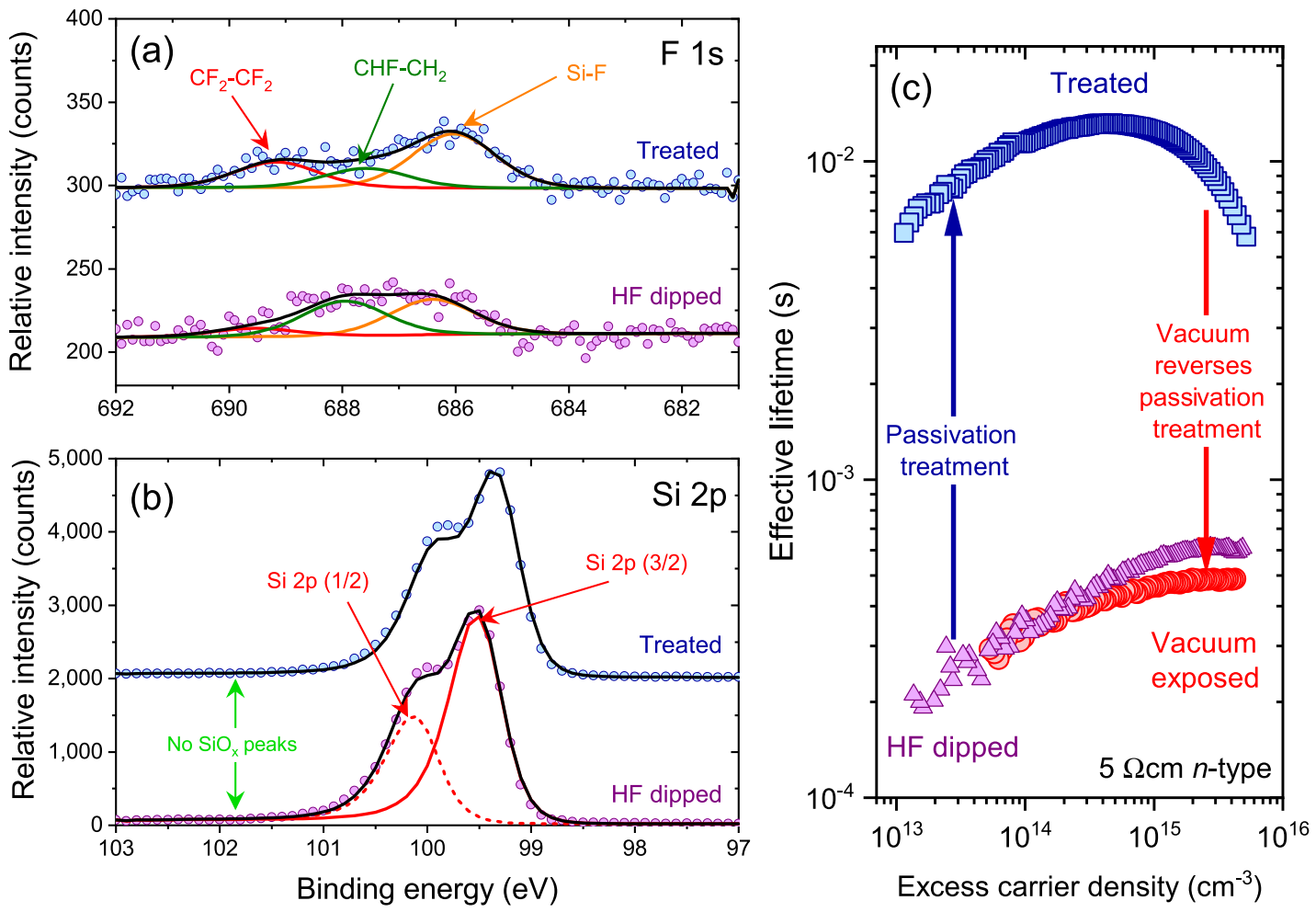

Figure 4. (a) XPS F 1s spectra for (NH)TFSI-hexane treated and HF-dipped Si samples, with the treated data shifted upward by 100 counts for clarity. (b) XPS Si 2p spectra for (NH)TFSI-hexane treated and HF-dipped samples, with the treated data shifted upward by 2000 counts for clarity. Both sets of XPS spectra are for a takeoff angle of $15^{\circ}$ to probe the near-surface region, with data for a takeoff angle of $90^{\circ}$ plotted in the Supporting Information (Figure S7). (c) Lifetime measurements performed in air for a sample after an HF dip, after passivation with (NH)TFSIhexane in the low specification glovebox, and after placing the passivated sample in a vacuum chamber.

surface potential of the treated surface becomes much less negative under illumination due to a reduction in the width of the depletion region. Similar behavior is found for $p$-type silicon (see Figure S3), although as $p$-type silicon is highly susceptible to bulk lifetime degradation, ${ }^{22} n$-type silicon provides a better controlled system for studying thin film passivation mechanisms. The KP results confirm the presence of a negative charge, similar to very recent work with Lewis acids. $^{23}$ As illustrated by the band diagrams, illumination generates electron-hole pairs in the silicon, which reduces the size of the depletion region and hence lowers the quasi-Fermi level for holes $\left(E_{\mathrm{Fp}}\right)$.

While stable enough for characterization purposes, the silicon surfaces passivated by TFSI-based solution are known to decay on the time scale of hours. ${ }^{4-6}$ The KP surface potential from a TFSI-hexane treated sample stored in air for $>20 \mathrm{~h}$ was monitored. The results in Figure $3 \mathrm{~b}$ show that the $\mathrm{KP}$ surface potential became less negative with time. After an initial increase, the relative KP surface potential increases fairly slowly before increasing more rapidly after $\sim 10 \mathrm{~h}$, prior to stabilization. The origin of the stabilization is currently not clear to us, but there are likely to be chemical changes in the film during the degradation and it possible that a very thin negatively charged film remains at the surface. Further data are presented in Figure S4, which provides simultaneous lifetime testing and relative $\mathrm{KP}$ surface potential measurements on two samples from the same wafer, but it is found that differences in the environment used for the different techniques have a strong impact on the degradation kinetics, as found previously. ${ }^{6}$ Photoconductance lifetime measurements made on the same sample as in Figure $3 \mathrm{~b}$ in the initial state and after
$20 \mathrm{~h}$ are shown in Figure 3c. While the lifetime reduces and the relative KP surface potential becomes less negative, detailed analysis of the injection dependence of the lifetime (Figure S5) shows that chemical passivation is likely to reduce too. Thus, while the film formed contains negative charge, the lifetime degradation is not just via reduction of charge in the external layer. This might occur by various mechanisms, including chemical changes (e.g., a reaction with water from the atmosphere), leakage of charge across the surface of the silicon, or accumulation of charged/polarizable species from the atmosphere which compensates the film's charge. Finally, it is noted that corona charging experiments were used in an attempt to modify the charge level in the passivating film, but either polarity of the corona charging rapidly $(<60 \mathrm{~s})$ resulted in a lifetime reduction (Figure S6). This is likely to be due to damage to the thin passivation layer from corona charge breakdown, as has been observed when an excessive electric field is applied to a dielectric-silicon system. ${ }^{24}$

To investigate the structure and chemical bonding at the silicon surface, X-ray photoelectron spectroscopy (XPS) was performed on TFSI-hexane treated silicon and just HF-dipped silicon. The shallow takeoff angle $\left(15^{\circ}\right)$ F 1 s and Si 2 p spectra are shown in Figure 4 panels a and b, respectively, with the $90^{\circ}$ takeoff angle data shown in the Supporting Information (Figure S7). Although XPS has been used on TFSI-treated silicon surfaces before, ${ }^{4}$ we need to be cautious in our interpretation as the passivation lacks vacuum stability. Figure $4 \mathrm{c}$ shows carrier lifetime for the same sample: (i) after an HF dip; then (ii) after TFSI-hexane treatment; then (iii) after being exposed to a short subsequent vacuum treatment $(<6 \times$ $10^{-5} \mathrm{mbar}$ ). The vacuum treatment reverses the effects of the 
(a) On droplet
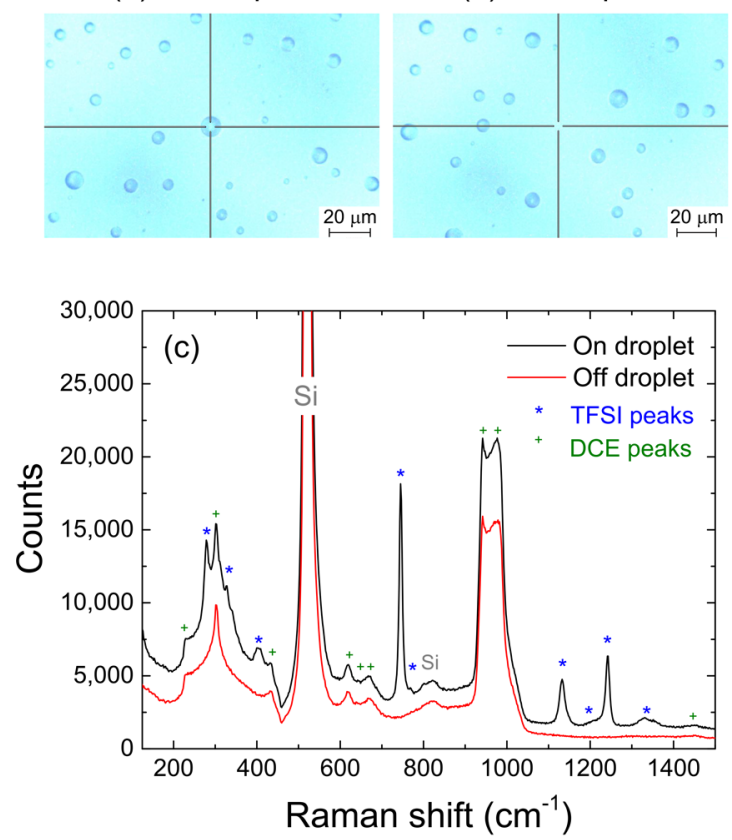

(d)

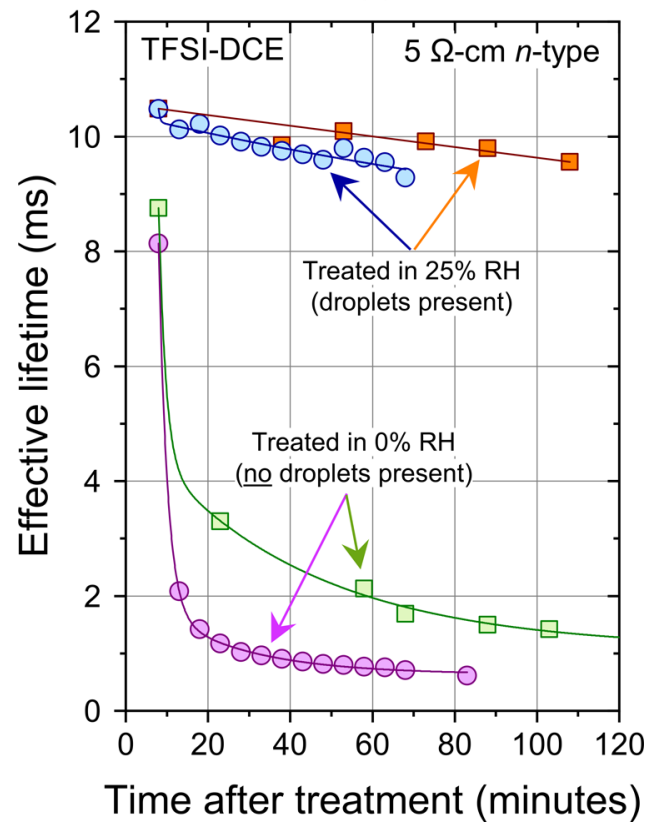

Figure 5. ( $a$ and $b$ ) Optical micrographs of droplets of passivation solution on a silicon surface after (NH)TFSI-DCE passivation treatment. (c) Raman spectra acquired from the droplet in panel a and in a region between the droplets in panel $b$. Details of the peak assignments are given in the Supporting Information (Table S1). (d) Effective lifetime measured versus time in air for samples treated with (NH)TFSI-DCE in 25\% RH (to have droplets present) and $0 \% \mathrm{RH}$ (to have no droplets present), with curves plotted to guide the eye.

treatment with the trifluoromethanesulfonyl-based solution, resulting in essentially the same lifetime as in the HF-dipped state, which is still substantially higher than for a non-hydrogen terminated surface. Furthermore, by performing the vacuum treatment in a chamber with an optical window, we were able to observe the disappearance of a film from the silicon's surface while also experiencing a simultaneous increase in chamber pressure. Our XPS measurements were made under an ultrahigh vacuum level (down to $1 \times 10^{-10} \mathrm{mbar}$ ) and consequently it seems probable that we are not able to measure the true properties of the passivating film with this technique.

With these limitations considered, the XPS data still provide some insight into the surface chemistry. First, the F 1s spectra in Figure 4a show evidence for $\mathrm{Si}-\mathrm{F}$ bonding at the surface. Signals related to $\mathrm{CF}_{2}-\mathrm{CF}_{2}$ and $\mathrm{CHF}-\mathrm{CH}_{2}$ are required to fit the data. The same three peaks are required to fit the $\mathrm{F} 1 \mathrm{~s}$ spectra for the HF-dipped and TFSI-hexane treated surfaces. This implies that the fluorine at the surface comes from the HF dip process (which is also performed before the TFSI-hexane treatment) and not necessarily from the fluorine-containing TFSI molecule. Second, the Si $2 p$ spectra in Figure $4 b$ show no evidence for $\mathrm{SiO}_{x}$ peaks at the silicon surface. As $\mathrm{SiO}_{x}$ layers are likely to be vacuum stable this shows that $\mathrm{SiO}_{x}$ layers, such as a native oxide, do not play a role in the passivation.

With the new data presented in this paper, we are able to propose a model for the passivation of surfaces by trifluoromethanesulfonyl-based solutions, including TFSI. Our results show there are at least two components to the passivation. The first is chemical passivation, similar to that which occurs for a hydrogen-terminated surface. HF-treated silicon surfaces are well-known to be hydrogen-terminated $^{12,25,26}$ and the HF pretreatment appears to provide a direct or indirect source of chemical passivation in combination with the trifluoromethanesulfonyl-based treatment. The second component is field effect passivation and this is proposed to arise from a negatively charged thin film on the surface. The reversibility in carrier lifetime shown by the vacuum exposure experiment (Figure 4c) implies that the film interacts with the surface by physical adsorption (physisorption), rather than chemisorption.

Interestingly, when a silicon sample is treated with a bis(trifluoromethanesulfonyl)-based solution we often observe circular droplets to remain on the surface (Figure 5a,b). The droplets are not observed when the treatment is performed in a low relative humidity $(\mathrm{RH})$ environment. When droplets are present they can be washed off with a solvent without reducing the initial carrier lifetime (hence surface passivation level) substantially. Lifetime data in Figure 5d show that similar high levels of initial passivation are achieved with and without droplets present, however the droplets play an important role in stabilizing the field effect passivation. As shown in Figure 5d, samples with droplets present retain their high lifetimes for well over an hour, whereas the lifetime in samples without droplets decays very rapidly. The droplets seem to protect the passivation from being degraded by the air environment, but over time the droplets themselves disappear, hence explaining the steady decay in passivation level. A speculative mechanism could be that the droplets act as a reservoir to replenish the thin film adsorbed at the surface, which evaporates upon air exposure. The degradation in KP surface potential with time (Figure $3 b$ ) would therefore be explained in terms of charge loss due to evaporation of the passivating film itself.

Finally, Raman spectroscopy was performed on a silicon sample treated with a bis(trifluoromethanesulfonyl)-based solution with the spectra plotted in Figure 5c. This study was performed using a TFSI-DCE solution as used in previous works. $^{4-6}$ The two spectra shown were acquired from the locations in Figure 5a,b, centering the acquisition area "on" a droplet and "off" a droplet, respectively. Many features are present in both spectra, with the large signal at $520 \mathrm{~cm}^{-1}$ 
arising from the silicon substrate and several peaks due to alkyl $(\mathrm{C}-\mathrm{Cl})$ and $(\mathrm{C}-\mathrm{C}-\mathrm{Cl})$ bond vibrations originating from the DCE solvent. ${ }^{27}$ Clear additional peaks arising from the droplet are found at 744,1132 , and $1242 \mathrm{~cm}^{-1}$, with less clear features observed at 279, 325, 402, 1206, and $1330 \mathrm{~cm}^{-1}$. All these peaks can be assigned to various deformation modes of the $\mathrm{CF}_{3}$ and $\mathrm{SO}_{2}$ groups in the TFSI anion. ${ }^{28,29}$ Peaks are also present in both spectra at $119,229,302,617,640,667,942$, 978 , and $1447 \mathrm{~cm}^{-1}$. These peaks can all be assigned to known vibration modes in DCE. ${ }^{27}$ In addition to the very intense bulk silicon vibration peak at $520 \mathrm{~cm}^{-1}$ found in both spectra as expected, a much weaker broad peak is observed around 821 $\mathrm{cm}^{-1}$ in both spectra. This is believed to be due to the HFbased cleaning procedure used on the silicon samples prior to the bis(trifluoromethanesulfonyl)-based solution treatment, as observed in Raman spectra taken from similar samples by Wang et al. ${ }^{30}$ and Ren et al.; ${ }^{31}$ however, it is not clear from this prior work exactly to what bond this peak should be attributed. Given the nature of the acid treatment it seems likely that an $\mathrm{Si}-\mathrm{H}$ or similar vibration is the cause. A complete list of peaks and their assignment to bond vibrations in silicon, DCE, and TFSI based on existing information in the literature is given in the Supporting Information (Table S1).

The presence of TFSI in the droplets is consistent with the NMR data which show TFSI does not initially dissociate in the solvent (Figure 2a,b). There are no additional peaks arising from the physisorbed thin film responsible for the field effect passivation, which suggests that the passivating film is very thin, and/or is made up of molecules and ions containing the same bonds as silicon, DCE, and TFSI. It is difficult to assess the thickness of the film from the Raman data without more knowledge of the composition of the film, given that Raman microspectroscopy has previously been used to detect polymer films of as little as $3 \mathrm{~nm}$ thickness and even monolayers and submonolayers of certain organic substances. ${ }^{32}$

\section{CONCLUSIONS}

In this study we have demonstrated that a range of solutions based on molecules with a bis(trifluoromethanesulfonyl)-based structure can give rise to excellent passivation of surfaces. While this includes the superacid TFSI, a high level of passivation also arises from solutions which are nonacidic, and so (super)acidity is not a prerequisite for the passivation. The surface passivation achieved has both a chemical and a field effect component. Chemical passivation arises at least partially from hydrogen termination of the surface. The solution treatment adds a field effect element being explainable by the existence of a negatively charged thin film physisorbed on the surface. This film is unstable in air and under vacuum, with the degradation in air being less severe when small droplets of solution help stabilize the film. The finding that nonacidic solutions offer similar levels of passivation as superacidic solutions means that these treatments could find future uses in materials systems sensitive to acidic environments, such as in perovskite solar cells.

\section{ASSOCIATED CONTENT}

\section{S Supporting Information}

The Supporting Information is available free of charge on the ACS Publications website at DOI: 10.1021/acsaelm.9b00251.

NMR degradation spectra over time, photographs of color changes in solutions, lifetime and KP data for a $p$ - type sample, further lifetime and KP time dependent data, fitting of injection dependent lifetime curves, lifetime data for corona-charged samples, XPS data with a $90^{\circ}$ takeoff angle, details of the Raman peak assignments (PDF)

\section{AUTHOR INFORMATION}

\section{Corresponding Author}

*E-mail: john.d.murphy@warwick.ac.uk. ORCID

John D. Murphy: 0000-0003-0993-5972

\section{Author Contributions}

\#Joint first authors. These authors contributed equally to the paper. A.I.P., N.E.G., P.R.W. and J.D.M. developed the ideas for the experiments. A.I.P. and N.E.G. performed lifetime measurements. R.S.B., A.I.P. and P.R.W. performed and interpreted KP experiments, with assistance from N.E.G. and J.D.M. A.I.P. and E.C.W.-J. performed NMR experiments. M.W. performed and interpreted the results of XPS experiments. C.E.J.D. interpreted the Raman spectra which were acquired by A.I.P. J.D.M. supervised the work and wrote the manuscript.

\section{Notes}

The authors declare no competing financial interest. Data published in this article can be freely downloaded from http://wrap.warwick.ac.uk/118640/.

\section{ACKNOWLEDGMENTS}

This work was funded by the EPSRC SuperSilicon PV project $(\mathrm{EP} / \mathrm{M} 024911 / 1)$ and the EPSRC Impact Acceleration Account (EP/R511808/1). A. I. Pointon is the recipient of an EPSRC studentship (EP/N509796/1), E. C. Wheeler-Jones was supported by the EPSRC Centre for Doctoral Training in Molecular Analytical Science (EP/L015307/1), and R. S. Bonilla held an ESPRC Postdoctoral Fellowship (EP/ M022196/1).

\section{REFERENCES}

(1) Amani, M.; Lien, D.-H.; Kiriya, D.; Xiao, J.; Azcatl, A.; Noh, J.; Madhvapathy, S. R.; Addou, R.; Kc, S.; Dubey, M.; Cho, K.; Wallace, R. M.; Lee, S.-C.; He, J.-R.; Ager III, J. W.; Zhang, X.; Yablonovitch, E.; Javey, A. Near-unity photoluminescence quantum yield in $\mathrm{MoS}_{2}$. Science 2015, 350, 1065-1068.

(2) Alemu, Y. A.; Albuquerque, G. H.; Herman, G. S. Enhanced photoluminescence from $\mathrm{CuInS}_{2} / \mathrm{ZnS}$ quantum dots: Organic superacid passivation. Mater. Lett. 2018, 219, 178-181.

(3) Piper, D. M.; Evans, T.; Leung, K.; Watkins, T.; Olson, J.; Kim, S. C.; Han, S. S.; Bhat, V.; Oh, K. H.; Buttry, D. A.; Lee, S.-H. Stable silicon-ionic liquid interface for next-generation lithium-ion batteries. Nat. Commun. 2015, 6, 6230.

(4) Bullock, J.; Kiriya, D.; Grant, N.; Azcatl, A.; Hettick, M.; Kho, T.; Phang, P.; Sio, H. C.; Yan, D.; Macdonald, D.; Quevedo-Lopez, M. A.; Wallace, R. M.; Cuevas, A.; Javey, A. Superacid Passivation of Crystalline Silicon Surfaces. ACS Appl. Mater. Interfaces 2016, 8, 24205-24211.

(5) Grant, N. E.; Niewelt, T.; Wilson, N. R.; Wheeler-Jones, E. C.; Bullock, J.; Al-Amin, M.; Schubert, M. C.; van Veen, A. C.; Javey, A.; Murphy, J. D. Superacid-Treated Silicon Surfaces: Extending the Limit of Carrier Lifetime for Photovoltaic Applications. IEEE Journal of Photovoltaics 2017, 7, 1574-1583.

(6) Pointon, A. I.; Grant, N. E.; Wheeler-Jones, E. C.; Altermatt, P. P.; Murphy, J. D. Superacid-derived surface passivation for measurement of ultra-long lifetimes in silicon photovoltaic materials. Sol. Energy Mater. Sol. Cells 2018, 183, 164-172. 
(7) Amani, M.; Taheri, P.; Addou, R.; Ahn, G. H.; Kiriya, D.; Lien, D.-H.; Ager, J. W.; Wallace, R. M.; Javey, A. Recombination Kinetics and Effects of Superacid Treatment in Sulfur- and Selenium-Based Transition Metal Dichalcogenides. Nano Lett. 2016, 16, 2786-2791.

(8) Grant, N. E.; Murphy, J. D. Temporary Surface Passivation for Characterisation of Bulk Defects in Silicon: A Review. Phys. Status Solidi RRL 2017, 11, 1700243.

(9) Suarez, S. N.; Jayakody, J. R. P.; Greenbaum, S. G.; Zawodzinski Jr, T.; Fontanella, J. J. A Fundamental Study of the Transport Properties of Aqueous Superacid Solutions. J. Phys. Chem. B 2010, 114, 8941-8947.

(10) Kiriya, D.; Hijikata, Y.; Pirillo, J.; Kitaura, R.; Murai, A.; Ashida, A.; Yoshimura, T.; Fujimura, N. Systematic Study of Photoluminescence Enhancement in Monolayer Molybdenum Disulfide by Acid Treatment. Langmuir 2018, 34, 10243-10249.

(11) Lu, H.; Kummel, A.; Robertson, J. Passivating the sulfur vacancy in monolayer $\mathrm{MoS}_{2}$. APL Mater. 2018, 6, 066104.

(12) Yablonovitch, E.; Allara, D. L.; Chang, C. C.; Gmitter, T.; Bright, T. B. Unusually Low Surface-Recombination Velocity on Silicon and Germanium Surfaces. Phys. Rev. Lett. 1986, 57, 249-252.

(13) Kim, H.; Lien, D.-H.; Amani, M.; Ager, J. W.; Javey, A. Highly Stable Near-Unity Photoluminescence Yield in Monolayer $\mathrm{MoS}_{2}$ by Fluoropolymer Encapsulation and Superacid Treatment. ACS Nano 2017, 11, 5179-5185.

(14) Abdi-Jalebi, M.; Andaji-Garmaroudi, Z.; Cacovich, S.; Stavrakas, C.; Philippe, B.; Richter, J. M.; Alsari, M.; Booker, E. P.; Hutter, E. M.; Pearson, A. J.; Lilliu, S.; Savenije, T. J.; Rensmo, H.; Divitini, G.; Ducati, C.; Friend, R. H.; Stranks, S. D. Maximizing and stabilizing luminescence from halide perovskites with potassium passivation. Nature 2018, 555, 497-501.

(15) Kessler, M.; Ohrdes, T.; Altermatt, P. P.; Brendel, R. The effect of sample edge recombination on the averaged injection-dependent carrier lifetime in silicon. J. Appl. Phys. 2012, 111, 054508.

(16) Baikie, I. D.; Mackenzie, S.; Estrup, P. J. Z.; Meyer, J. A. Noise and the Kelvin method. Rev. Sci. Instrum. 1991, 62, 1326-1332.

(17) Alharbi, A.; Zahl, P.; Shahrjerdi, D. Material and device properties of superacid-treated monolayer molybdenum disulfide. Appl. Phys. Lett. 2017, 110, 033503.

(18) Yu, Y.; Li, G.; Huang, L.; Barrette, A.; Cai, Y.-Q.; Yu, Y.; Gundogdu, K.; Zhang, Y.-W.; Cao, L. Enhancing Multifunctionalities of Transition-Metal Dichalcogenide Monolayers via Cation Intercalation. ACS Nano 2017, 11, 9390-9396.

(19) Dolbier, W. R. Guide to fluorine NMR for organic chemists.; Wiley: 2009.

(20) Foropoulos, J., Jr.; DesMarteau, D. D. Synthesis, properties, and reactions of bis $($ (trifluoromethyl $)$ sulfonyl $)$ imide, $\left(\mathrm{CF}_{3} \mathrm{SO}_{2}\right)_{2} \mathrm{NH}$. Inorg. Chem. 1984, 23, 3720-3723.

(21) Markevich, E.; Sharabi, R.; Borgel, V.; Gottlieb, H.; Salitra, G.; Aurbach, D.; Semrau, G.; Schmidt, M. A. In situ FTIR study of the decomposition of N-butyl-N-methylpyrrolidinium bis(trifluoromethanesulfonyl)amide ionic liquid during cathodic polarization of lithium and graphite electrodes. Electrochim. Acta 2010, 55, $2687-2696$.

(22) Niewelt, T.; Selinger, M.; Grant, N. E.; Kwapil, W. M.; Murphy, J. D.; Schubert, M. C. Light-induced activation and deactivation of bulk defects in boron-doped float-zone silicon. J. Appl. Phys. 2017, $121,185702$.

(23) Ji, W.; Zhao, Y.; Fahad, H. M.; Bullock, J.; Allen, T.; Lien, D.H.; De Wolf, S.; Javey, A. Dip Coating Passivation of Crystalline Silicon by Lewis Acids. ACS Nano 2019, 13, 3723-3729.

(24) Bonilla, R. S.; Reichel, C.; Hermle, M.; Hamer, P.; Wilshaw, P. $\mathrm{R}$. Long term stability of $\mathrm{c}-\mathrm{Si}$ surface passivation using corona charged $\mathrm{SiO}_{2}$. Appl. Surf. Sci. 2017, 412, 657-667.

(25) Burrows, V. A.; Chabal, Y. J.; Higashi, G. S.; Raghavachari, K.; Christman, S. B. Infrared spectroscopy of $\mathrm{Si}(111)$ surfaces after HF treatment: Hydrogen termination and surface morphology. Appl. Phys. Lett. 1988, 53, 998-1000.
(26) Fenner, D. B.; Biegelsen, D. K.; Bringans, R. D. Silicon surface passivation by hydrogen termination: A comparative study of preparation methods. J. Appl. Phys. 1989, 66, 419-424.

(27) Mizushima, S.; Shimanouchi, T.; Harada, I.; Abe, Y.; Takeuchi, $\mathrm{H}$. Infrared and Raman Spectra of 1,2-Dichloroethane and its Deuterium Compound in the Gaseous, Liquid, and Solid States. Can. J. Phys. 1975, 53, 2085-2094.

(28) Rey, L.; Johansson, P.; Lindgren, J.; Lassegues, J. C.; Grondin, J.; Servant, L. Spectroscopic and Theoretical Study of $\left(\mathrm{CF}_{3} \mathrm{SO}_{2}\right)_{2} \mathrm{~N}^{-}$ $\left(\mathrm{TFSI}^{-}\right)$and $\left(\mathrm{CF}_{3} \mathrm{SO}_{2}\right)_{2} \mathrm{NH}$ (HTFSI). J. Phys. Chem. A 1998, 102, 3249-3258

(29) Hardwick, L. J.; Holzapfel, M.; Wokaun, A.; Novák, P. Raman study of lithium coordination in EMI-TFSI additive systems as lithium-ion battery ionic liquid electrolytes. J. Raman Spectrosc. 2007, $38,110-112$.

(30) Wang, J.; Tu, H.; Zhu, W.; Zhou, Q.; Liu, A.; Zhang, C. A comparative Raman spectroscopy study on silicon surface in HF, $\mathrm{HF}: \mathrm{H}_{2} \mathrm{O}_{2}$ and HF: $\mathrm{NH}_{4} \mathrm{~F}$ aqueous solutions. Mater. Sci. Eng., B 2000, 72, 193-196

(31) Ren, B.; Liu, F. M.; Xie, J.; Mao, B. W.; Zu, Y. B.; Tian, Z. Q. In situ monitoring of Raman scattering and photoluminescence from silicon surfaces in HF aqueous solutions. Appl. Phys. Lett. 1998, 72, 933-935.

(32) Liszka, B. M.; Lenferink, A. T. M.; Witkamp, G.-J.; Otto, C. Raman micro-spectroscopy for quantitative thickness measurement of nanometer thin polymer films. J. Raman Spectrosc. 2015, 46, 12301234. 